Journal of Computer Science 5 (6): 445-450, 2009

ISSN 1549-3636

(C) 2009 Science Publications

\title{
Analytic Performability Model of Vertical Handoff in Wireless Networks
}

\author{
${ }^{1}$ Gowrishankar, ${ }^{2}$ G.N. Sekhar and ${ }^{3}$ P.S. Satyanarayana \\ ${ }^{1}$ Department of Computer Science and Engineering, BM Sreenivasaiah College of Engineering, \\ Visvesvaraya Technological University, Bangalore-560019, India \\ ${ }^{2}$ Department of Mathematics, B.M.Sreenivasaiah College of Engineering, \\ Visvesvaraya Technological University, Bangalore-560019, India \\ ${ }^{3}$ Department of Electronics and Communication Engineering, \\ BM Sreenivasaiah College of Engineering, \\ Visvesvaraya Technological University, Bangalore-560019, India
}

\begin{abstract}
Problem statement: The next generation wireless systems should be designed to support heterogeneous traffic with seamless mobility. A single network alone cannot cope up with such heterogeneous requirements. Hence it is desirable to interoperate between diverse and complementary Radio Access Technologies (RATs). In such system, user will switch between different Radio Access Technologies (RATs) to satisfy the User/application requirement and this process is known as Vertical Handoff (VHO). The process of network switching had three phases, network discovery, handoff decision and execution. The decision phase played a crucial role in resource utilization and user/application Quality of Service (QoS) requirement. Hence it was essential to model and evaluate the handoff decision system along with VHO system model. Approach: The traditional performance models were optimistic models and would evaluate the system performance under ideal condition by ignoring failures and recovery in the system. The availability models were conservative models and would assess the availability/reliability of the system. The performabality models were combined models of performance and reliability. The performablity models were more realistic models of the system due to the simultaneous consideration of performance and reliability. Here the VHO process of a next generation wireless system was modeled and evaluated by an analytic performablity model and performance of decision system is evaluated through the sensitivity analysis of VHO decision parameter. Results: In VHO performance evaluation, the metrics of performance evaluation are handoff dropping probability and new call blocking probability. The dynamics of these metrics are depends on set of wireless network parameter such as Available Bandwidth (ABW), users, Bit Error Rate (BER) and network traffic. The ABW, BER and network traffic is also parameter for VHO decisions. The results of performance evaluation are used to develop a novel intelligent vertical handoff decision technique to achieve optimum tradeoff between set of handoff decision criteria. Finally, sensitivity analysis of system parameters on four traffic classes and two vertical handoff decision algorithms along with intelligent vertical handoff decision method were presented. Conclusion: The results of sensitivity analysis depicted that the VHO process in next generation wireless system needs intelligent criteria based technique at the decision making phase of VHO process.
\end{abstract}

Key words: Radio access technologies, vertical handoff, quality of service, multi criteria decision making and artificial neural network

\section{INTRODUCTION}

The next generation wireless systems should support diverge network requirements of application and user with seamless mobility. The single wireless system alone cannot cope up with such requirements of traffic and user with ubiquitous network access. In this scenario next generation wireless systems will allow the user/mobile terminal to freely roam among set of complementary RATs to satisfy the application and user QoS requirements. The process of roaming will involve switching of user and application session

Corresponding Author: Gowrishankar, Department of Computer Science and Engineering,

B.M. Sreenivasaiah College of Engineering, Visvesvaraya Technological University,

Bangalore-560019, India 


\section{J. Computer Sci., 5 (6):445-450, 2009}

between two complementary RATs and this is called as vertical handoff. The vertical handoff has three phases: network discovery, handoff decision and handoff execution $^{[1]}$. The decision phase will play an important role in the user/application performance and system performance. The user performance metrics are handoff dropping and call blocking probability. The system performance metric is network utilization. Thus there is a need of efficient decision mechanism in meeting QoS requirement of user/application and optimum utilization of network resource. In order to design the efficient handoff system the designer needs to have deep insight about the dynamics of handoff metrics and the set of system parameters that influence on the dynamics of these metrics. In this scenario it is necessary to develop a realistic performance model to understand the behavior of handoff decision system.

The traditional performance models are optimistic models and will evaluate the system performance under ideal conditions by ignoring the failures and recovery in the system. The pure availability models are conservative models and will access the availability/reliability of the system ${ }^{[2]}$. The performability model is a combined model of performance and reliability/availability ${ }^{[3]}$. In performability model there is a simultaneous consideration of both performance and availability measures ${ }^{[4]}$. Hence there is a need to develop performability model for vertical handoff decision system. The performability model parameters are same as handoff decision system parameters such as ABW, BER and traffic ${ }^{[5]}$. The results of performability models are used to develop an intelligent handoff decision system. The sensitivity analysis of handoff decision system are carried out on four different class wireless traffic such as conversational, streaming, interactive and background. To validate the responsiveness of the intelligent handoff decision system, the decision system is also compared with Simple Additive Weights (SAW) and Technique of Order of Preference by Similarity to Ideal Solution (TOPSIS) handoff decision algorithms ${ }^{[6]}$, the results obtained from proposed system are promising.

The early works of performance evaluation is mostly on homogenous handoff system either on pure performance of the handoff system or on availability/reliability model of the wireless network $^{[2,3]}$. Some works were also reported on combined model and such models are called as Performability model ${ }^{[4]}$. But all these performance evaluation technique is limited to horizontal handoff mechanism. In the field of vertical handoff performance evaluation, few works were reported on handoff decision metrics such as average power consumption and average user preference dissatisfaction. But these metrics are specific to a vertical handoff decision algorithm $^{[7,8]}$. A work was also reported on performance evaluation of vertical handoff system with four different decision algorithm with respect to bandwidth allocation and average delay of each flow of individual algorithm with an ideal wireless environment ${ }^{[9]} . \operatorname{In}^{[10]}$, sensitivity analyses of handoff parameters are considered for a delay sensitive traffic class, in an ideal wireless environment. In this research the Performability models were developed to analyze the behavior of vertical handoff decision system under a realistic wireless environment

Performability model: The Performability model is divided in to pure performance model and availability model. The system under study is having three set of users and coverage area of each RAT is known as cell. The first set of users is new users to the system and their network service request is originated from the same cell. The second set of users are those users whose network service request is originated from different cell of analogous RAT but their mobility is restricted to different cell of the similar RAT. These users are called as horizontal handoff users. The last and third sets of users are vertical handoff users and their mobile terminals are equipped with heterogeneous RAT access technology. In this system the network ABW is divided in to set of logical or virtual channels ${ }^{[1]}$ and these logical channels are shared among set of users. The concept of guard channels are used to improve the handoff performance in the system ${ }^{[3]}$. Here set of performance metrics are vertical handoff dropping probability $\mathrm{P}_{\mathrm{VD}}$, horizontal handoff dropping probability $\mathrm{P}_{\mathrm{HD}}$ and new user blocking probability $\mathrm{P}_{\mathrm{ND}}$.

Performance model: Here, pure performance assessment of the handoff decision system is presented. The new user to the system will originate network request at the rate $\lambda_{\mathrm{N}}$ and is assumed to follow a Poisson process. The horizontal handoff requests to the designated cell will arrive at the rate $\lambda_{\mathrm{H}}$ and it is assumed to be a Poisson process. Vertical handoff requests to the designated cell are at the rate $\lambda_{\mathrm{V}}$ and will follow the Poisson Process. The mean service time of all types of users were assumed to follow negative exponential distribution with the mean rate $1 / \mu$. The total number of virtual channel in the system are $\mathrm{N}$. When the numbers of available channels are below the specified threshold in the system will accept only horizontal and vertical handoff request. The threshold limit is determined by two positive integers $\mathrm{C}_{1}$ and $\mathrm{C}_{2}$. When the available 
number of channels falls below the threshold $\mathrm{C}_{2}$ the proposed system will accept only vertical handoff request. The vertical handoff users are given highest priority than the other two types of users. The Markov chain can be formulated for the above description. $\mathrm{In}^{[12]}$, detail analysis of Markov chains are presented, under steady state condition $\mathrm{P}_{\mathrm{VD}}, \mathrm{P}_{\mathrm{HD}}$ and $\mathrm{P}_{\mathrm{ND}}$ are given by:

$$
\begin{aligned}
& P_{V D}(N)=\frac{\frac{A^{c_{1}} A_{1}^{c_{2}} A_{2}^{N-\left(c_{1}+c_{2}\right)}}{N !}}{\sum_{j=0}^{c_{1}} \frac{A^{j}}{j !}+\sum_{j=c_{1}+1}^{c_{2}} \frac{A^{c_{1}} A_{1}^{j-c_{1}}}{j !}+\sum_{j=c_{2}+1}^{N} \frac{A^{c_{1}} A_{1}^{c_{2}} A_{2}^{j-\left(c_{1}+c_{2}\right)}}{j !}} \\
& P_{H D}(N)=\frac{\sum_{i=c_{2}}^{N} \frac{A^{c_{1}} A_{1}^{c_{2}} A_{2}^{i-\left(c_{1}+c_{2}\right)}}{i !}}{\sum_{j=0}^{c_{1}} \frac{A^{j}}{j !}+\sum_{j=c_{1}+1}^{c_{2}} \frac{A^{c_{1}} A_{1}^{j-c_{1}}}{j !}+\sum_{j=c_{2}+1}^{N} \frac{A^{c_{1}} A_{1}^{c_{2}} A_{2}^{j-\left(c_{1}+c_{2}\right)}}{j !}} \\
& P_{N D}(N)=\frac{\sum_{i=c_{1}}^{c_{2}} \frac{A^{c_{1}} A_{1}^{i-c_{1}}}{i !}+\sum_{i=c_{2}+1}^{N} \frac{A^{c_{1}} A_{1}^{c_{2}} A_{2}^{N-\left(c_{1}+c_{2}\right)}}{i !}}{\sum_{j=0}^{c_{1}} \frac{A^{j}}{j !}+\sum_{j=c_{1}+1}^{c_{2}} \frac{A^{c_{1}} A_{1}^{j-c_{1}}}{j !}+\sum_{j=c_{2}+1}^{N} \frac{A^{c_{1}} A_{1}^{c_{2}} A_{2}^{j-\left(c_{1}+c_{2}\right)}}{j !}}
\end{aligned}
$$

Availability model: The availability model of the proposed system is when all $\mathrm{N}$ channels are failed then the system is unavailable for data transmission. Here the system is modeled as independent failure-repair model. Each virtual channel is subjected to time and frequency selective fading and multipath fading then the virtual channel will be unavailable for data transmission. The individual channel is available for the use by changing the mobile terminal position or by channel equalization technique ${ }^{[13]}$. The channel recovery is modeled as independent repair facility ${ }^{[14]}$. The failure rate of the channel is Poisson distribution with the rate $\gamma$. The channel recovery is exponential repair time distribution of parameter distribution with the parameter $\tau$. The $\mathrm{N}$ independent channel failure and recovery can be represented as a single dimension Markov Chain. The system availability can be modeled as Markov chain. The steady state probability $\mathrm{P}(\mathrm{i})$, where $\mathrm{i}$ is the number of available channel in the system and given by:

$$
P(i)=\frac{\left(\frac{\tau}{v}\right)^{i} i !}{\prod_{j=0}^{i-1}(N-j)} \times\left(\sum_{i=0}^{N} \frac{\left(\frac{\tau}{v}\right)^{i} i !}{\prod_{j=0}^{i-1}(N-j)}\right)^{-1}
$$

When $\mathrm{K}$ is the number of available channel the system in the Eq. 3-5 the parameter $\mathrm{N}$ will be replaced by $\mathrm{K}$ and resulting equations are the realistic performance equation in the independent virtual channel failure and recovery scenario. The parameter values of Performability model are $\mathrm{N}=50, \mathrm{G}_{1}=20$, $\mathrm{G}_{2}=0-10, \lambda=7, \lambda_{1}=3, \lambda_{2}=1, \mu=2, \gamma=3$ and $\tau=2$. Figure 1 shows variation of $\mathrm{P}_{\mathrm{VD}}$ and $\mathrm{P}_{\mathrm{HD}}$ against number of guard channels.

The important observations from Performability model are: Increase in the number of vertical handoff guard channels will reduce the $\mathrm{P}_{\mathrm{VD}}$ but will increase the $\mathrm{P}_{\mathrm{HD}}$. Decreasing the number of vertical handoff guard channels will increase the $P_{V D}$ but will decrease the $\mathrm{P}_{\mathrm{HD}}$. The validity of the observations can be made from the Fig. 1.

In a next generation wireless system, it is desirable to maintain guaranteed QoS to the all types of users and optimum utilization of system resource needed. In order to provide better QoS to the user/application it is required to maintain $\mathrm{P}_{\mathrm{VD}}, \mathrm{P}_{\mathrm{HD}}$ and $\mathrm{P}_{\mathrm{ND}}$ in an acceptable limit. The bandwidth reservation scheme may reduce $P_{V D}$ but at the same time excessive bandwidth reservation will increase $\mathrm{P}_{\mathrm{HD}}, \mathrm{P}_{\mathrm{ND}}$ and under utilization of network bandwidth. Since all these are contradictory requirements and they cannot be satisfied simultaneously, it is desirable to use a multi constraint optimization technique in achieving better tradeoff between set of system performance metrics. The performance parameters such as BER, $\mathrm{ABW}$ and network traffic are the input to the optimization technique for achieving better tradeoff between set of performance metrics. Some of the well known techniques in solving multi constraint optimization problem are Game theory, Markov Decision Process (MDP), Genetic Algorithm (GA), Goal programming and Multi Criteria Decision Making (MCDM) technique. Among these set of techniques MCDM is a most widely used method ${ }^{[6]}$.

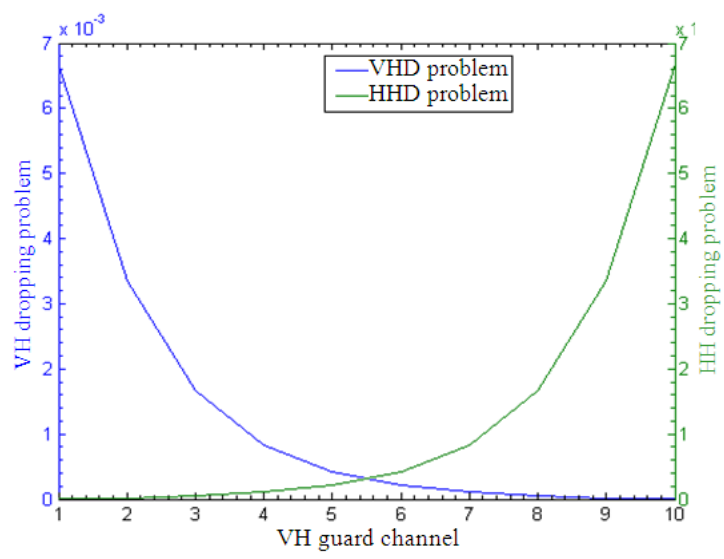

Fig. 1: $\mathrm{P}_{\mathrm{VD}}$ and $\mathrm{P}_{\mathrm{HD}} \mathrm{V} / \mathrm{s} \mathrm{VH}$ guard channel 


\section{MATERIALS AND METHODS}

Vertical handoff decision methods: The handoff decision process for network selection system is a multi constraint based multi criteria decision system. The MCDM systems are classified in to conventional and evolutionary methods. The common conventional methods are Analytic Hierarchical Process (AHP), Grey Relational Method (GRA), Multiplicative Exponent weighting (MEW), TOPSIS and SAW. The evolutionary approaches are Genetic Algorithm (GA), fuzzy logic and Artificial Neural Networks (ANN) ${ }^{[15]}$. In this study for the performance evaluation two algorithms from conventional method such as SAW and TOPSIS and neural network method from evolutionary approach is considered.

The SAW is the best known and most widely used scoring MCDM method ${ }^{[6]}$, a score in the SAW method is obtained by adding the contribution from each attribute or parameters. Since two items with different measurement unit cannot be added, a common numerical scaling of each attribute is required and the process of scaling the attributes is known as normalization. For the normalized attributes based on its importance the weights are assigned. The score for each alternative is obtained by multiplying the weights to the each normalized value of attributes. Formally value of an alternative in the SAW method can be expressed as:

$\mathrm{v}_{\mathrm{i}}=\sum_{\mathrm{j}=1}^{\mathrm{n}} \mathrm{w}_{\mathrm{j}} \mathrm{r}_{\mathrm{ij}}, \mathrm{i}=1, \ldots . ., \mathrm{m}$

Where:

$\mathrm{v}_{\mathrm{i}}=$ The score of each alternative

$\mathrm{w}_{\mathrm{j}}=$ The weight of each attribute

$r_{i j}=$ The normalized value of each parameter

In TOPSIS method with $\mathrm{m}$ alternatives that are evaluated by $\mathrm{n}$ parameters is viewed as a geometric system with $\mathrm{m}$ points in the $\mathrm{n}$ dimensional space. Here chosen alternative should have shortest distance to the ideal solution and longest distance to the negative ideal solution $^{[6]}$.

The application of ANN to solve complex decision problem is not very new and in particular to solve MCDM problem for crisp attributes the feed-forward ANN is used ${ }^{[16]}$. For fuzzy and fuzzy linguistic attribute values of MCDM system are explored using fuzzy Radial Basis Function (F-RBF) ANN and WDN (weighted Data Normalization)-RBF $\mathrm{ANN}^{[17,18]}$. No work is reported on solving MCDM problem of vertical handoff decision system with ANN method. Here F-RBF method is proposed for vertical handoff decision.

\section{RESULTS}

Performance evaluation: Here, performance evaluation of three different MCDM algorithms with four complementary overlaid RATs is presented. The attributes/criteria used for RAT selection are BER, $\mathrm{ABW}$, traffic, coverage/seamlessness and cost. The four attributes such as BER, ABW, traffic and cost are having crisp values and the coverage attribute having a fuzzy linguistic value. The fuzzy linguistic attribute is transformed in to crisp number according to standard rules $^{[17]}$. Table 1 presents characteristics of four RATs.

The sensitivity analysis for different weight for attribute value for four different traffic classes: Interactive, conversation, streaming and background traffic is shown in Fig. 2-5 respectively.

\begin{tabular}{lllllll}
\multicolumn{2}{l}{ Table 1: Characteristics of RATs } \\
$\begin{array}{lllllll}\text { Network } \\
\text { number }\end{array}$ & $\begin{array}{l}\text { Network } \\
\text { type }\end{array}$ & BER & $\begin{array}{l}\text { Traffic } \\
\text { (Mbps) }\end{array}$ & Coverage & $\begin{array}{l}\text { ABW } \\
\text { (Mbps) }\end{array}$ & Cost \\
\hline N-1 & $802.11 \mathrm{~b}$ & $10^{-3}$ & 2.2 & 0.717 & 8.8 & 1 \\
N-2 & $802.11 \mathrm{~b}$ & $10^{-3}$ & 4.4 & 0.500 & 6.6 & 1 \\
N-3 & $802.16 \mathrm{e}$ & $10^{-6}$ & 8.0 & 0.909 & 15.0 & 2 \\
N-4 & $802.11 \mathrm{a}$ & $10^{-5}$ & 32.4 & 0.283 & 21.6 & 2 \\
\hline
\end{tabular}

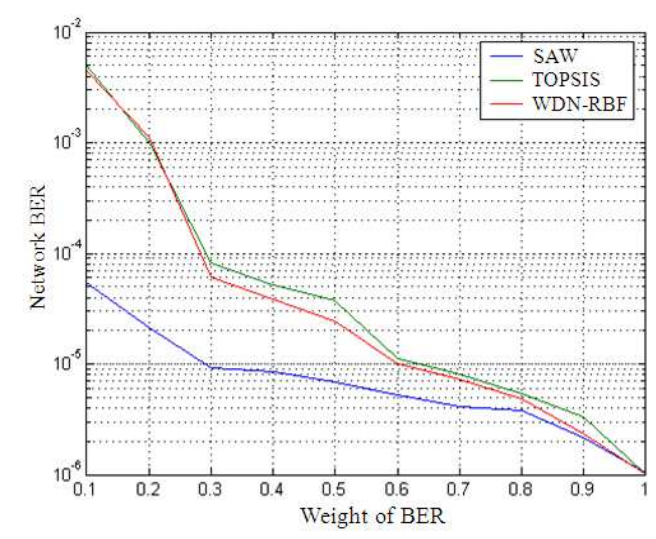

Fig. 2: Interactive traffic sensitivity to BER weight

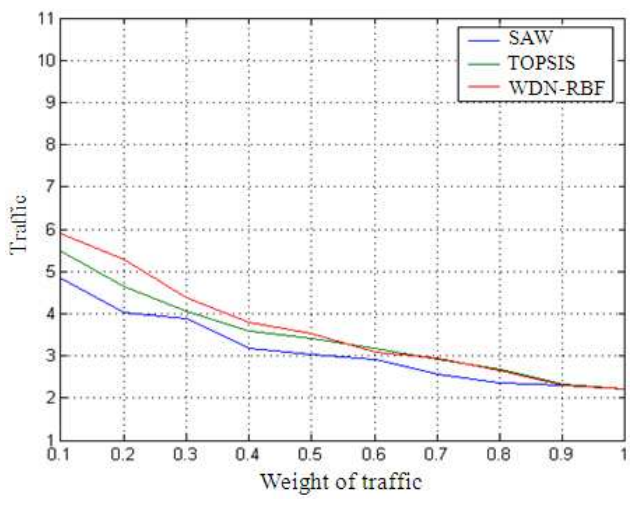

Fig. 3: Conversation traffic sensitivity to traffic weight 


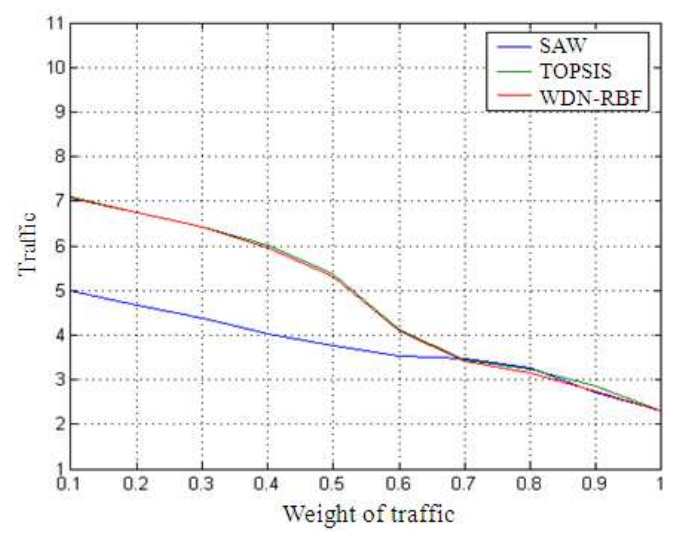

Fig. 4: Streaming traffic sensitivity to traffic weight

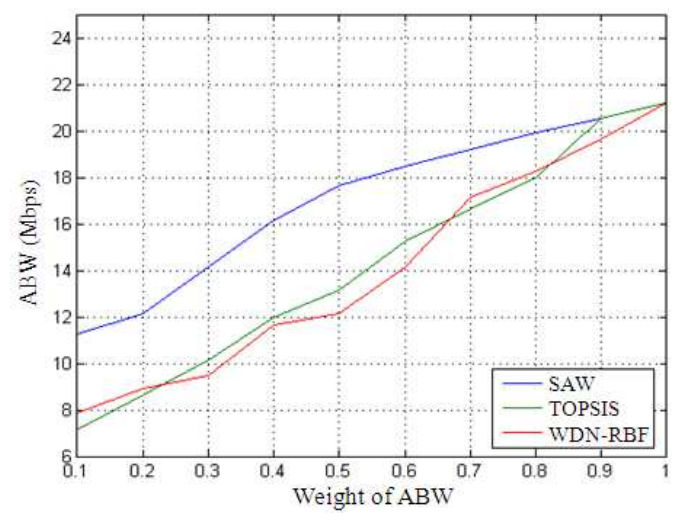

Fig. 5: Background traffic sensitivity to $\mathrm{ABW}$ weight

\section{DISCUSSION}

In this study analytic performablity model VHO along with performance evaluation of VHO decision system is presented. First performability model of vertical handoff decision system is illustrated. The results of performability model along with handoff decision system parameters are used to develop ANN based MCDM system for vertical handoff decision. The sensitivity analysis is conducted by varying the weights of different parameters of MCDM system for four types of traffic.

\section{CONCLUSION}

The observations from performance evaluation and sensitivity analysis are: interactive traffic is sensitive to the BER, the streaming and conversation traffic is sensitive to the network traffic and background traffic is sensitive to the ABW. The SAW MCDM is less sensitive to change in attribute weights than TOPSIS and WDN-RBF. The TOPSIS mechanism is stable and responsive to the variation of attribute weights but the numerical calculations make it prohibitive to use TOPSIS for online decision system. The vertical handoff decision mechanism requires online MCDM system The SAW and WDN-RBF are the ideal system for online decision making.

\section{REFERENCES}

1. Wen-Tsuen, C., L. Jen-Chu and H. Hsieh-kauan, 2004. An adaptive scheme for vertical handoffs in wireless overlay networks. Proceeding of the 10th International Conference on Parallel and Distributed Systems, July 7-9, IEEE Xplore Press, USA., $\quad$ pp: 541-548. DOI: 10.1109/ICPADS.2004.1316136

2. Ma, Y., J.J. Han and K.S. trevedi, 2001. Composite performance and availability analysis of wireless communication networks. IEEE Trans. Vehic. Technol., 50: 1216- $1223 . \quad$ DOI: $10.1109 / 25.950322$

3. Ma, Y., J.J. Han and K.S. Trevedi, 2000. Channel allocation and recovery strategy in wireless network. Eur. Trans. Telecommun., 11: 395-406. http://cat.inist.fr/?aModele $=$ afficheN\&cpsidt $=1441$ 147

4. Trivedi, K.S., X. Ma and S. Dharmaraja, 2003. Performablity model on wireless communication systems. J. Commun. Syst., 16: 561-577. DOI: 10.1002/dac.605

5. Song, Q. and A. Jamalipour, A quality of service negotiation-based vertical handoff decision scheme in heterogeneous wireless systems. Eur. J. Operat. Res., 191: 1059-1074. http://ideas.repec.org/a/eee/ejores/v191y2008i3p10 59-1074.html

6. Yoon, K.P. and C. Hwang, 1995. Multiple Attribute Decision Making. 1st Edn., Sage Publication, ISBN: 10: 0803954867, pp: 83.

7. Xing, B. and N. Venkatasubramanian, 2005. Multiconstraint dynamic acess selection in always best connected networks. Proceeding of the 2nd Annual International Conference on Mobile and Ubiquitous Systems: Networking and Services, July 17-21, IEEE Xplore Press, USA., pp: 56-64. DOI: 10.1109/MOBIQUITOUS.2005.39

8. Zhu, F. and J. McNair, 2006. Multiservice vertical handoff decision algorithms. EURASIP. J. Wireless Commun. Network., 2006: 52-52. http://portal.acm.org/citation.cfm?id=1283576 
9. Stevens-Navarro, E. and V.W.S. Wong, 2006. Comparison between vertical handoff decision algorithms for heterogeneous wireless networks. Proceeding of the IEEE Conference on Vehicular Technology, May 7-10, IEEE Xplore Press, Melbourne, Vic., pp: 947-951. DOI: 10.1109/VETECS.2006.1682964

10. Lassoued, I., J. Bonnin, Z.B. Hamoud and A. Belghith, 2008. A methodology for evaluating vertical handoff decision Mechanisms. Proceeding of the 7th International Conference on Networking, Apr. 13-18, IEEE Xplore Press, Cancun, pp: 377-384. DOI: $10.1109 / \mathrm{ICN} .2008 .71$

11. Janeveski, T., 2003. Traffic analysis and design of wireless IP networks. Artech. House Inc., MA., ISBN:1580533310, pp: 390.

12. Gowrishankar, H.S. Rameshbabu, G.T. Raju and P.S. Satyanarayana, 2008. Performability model of vertical handoff in wireless data networks. Proceedings of the 4th International Conference on Wireless and Mobile Communications-Volume 00, July 27-Aug. 01, IEEE Computer Society, Washington, DC., USA., pp: 309-314. http://portal.acm.org/citation.cfm?id=1448713

13. Trevedi, K.S., 2001. Probability and Statistics with Reliability, Queuing and Computer Science Application. 2nd Edn., John Wiley and Sons, ISBN: 10: 0471333417, pp: 830.
14. Mulgrw, B., 1996. Applying radial basis functions. IEEE Signal Process. Mag., 13: 50-65. DOI: 10.1109/79.487041

15. Marler, R.T. and J.S. Arora, 2004. Survey of multiobjective optimization methods for engineering. Struct. Multidisciplin. Optimiz., 26: 369-395. DOI: 10.1007/s00158-003-0368-6

16. Wang, J. and B. Malakooti, 1992. A feedforword neural networks for multiple criteria decision making. Comput. Operat. Res., 19: 151-167. http://portal.acm.org/citation.cfm?id=147830

17. Kong, F. and H. Liu, 2006. Fuzzy RBF neural network model for multiple attribute decision making. Lecture Notes Comput. Sci., 4234: 1046-1054. DOI: 10.1007/11893295_115

18. Song, Q. and N. Kasbov, 2004. WDN-RBF: Weighted data normalization for radial basis neural networks. Proceeding of the IEEE International Joint Conference on Neural Networks, July 25-29, Budapest, Hungary, pp: 2095-2098. http://cat.inist.fr/?aModele $=$ afficheN\&cpsidt $=1762$ 3781 